\title{
On the irreducibility of the two variable zeta-function for curves over finite fields
}

\author{
Niko Naumann
}

October 30, 2018

\begin{abstract}
In [P] R. Pellikaan introduced a two variable zeta-function $Z(t, u)$ for a curve over a finite field $\mathbb{F}_{q}$ which, for $u=q$, specializes to the usual zeta-function and he proved, among other things, rationality: $Z(t, u)=(1-t)^{-1}(1-u t)^{-1} P(t, u)$ with $P(t, u) \in \mathbb{Z}[t, u]$. We prove that $P(t, u)$ is absolutely irreducible. This is motivated by a question of J. Lagarias and E. Rains about an analogous two variable zetafunction for number fields.
\end{abstract}

MSC2000: 11G20 (primary), 14G10 (secondary)

\section{Introduction}

Let $X$ be a proper, smooth, geometrically connected curve of genus $g$ over the finite field $\mathbb{F}_{q}$. The zeta-function of $X / \mathbb{F}_{q}$ can be written as a power series

$$
Z(t)=\sum_{D} \frac{q^{h^{0}(D)}-1}{q-1} t^{\operatorname{deg}(D)} .
$$

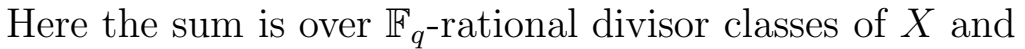
$h^{0}(D):=\operatorname{dim}_{\mathbb{F}_{q}} H^{0}(X, \mathcal{O}(D))$. Writing $b_{n k}$ for the number of divisor classes 
of degree $n$ and with $h^{0}(D)=k$ this becomes

$$
Z(t)=\sum_{n \geq 0} \sum_{k \geq 1} b_{n k} \frac{q^{k}-1}{q-1} t^{n} .
$$

In $[\mathrm{P}] R$. Pellikaan observed that the classical proof of rationality and the functional equation for $Z(t)$ go through when $q$ is treated as a variable in this expression. He thus introduced the following power series in $[\mathbb{P}]$, Def. 3.1:

$$
Z(t, u):=\sum_{n \geq 0} \sum_{k \geq 1} b_{n k} \frac{u^{k}-1}{u-1} t^{n}
$$

This is called the two variable zeta-function of the curve. We will denote by $h$ the class-number of $X / \mathbb{F}_{q}$, i.e. $h=\left|\operatorname{Pic}^{0}(X)\right|$. Then Pellikaan proved:

Theorem 1 We have

$$
Z(t, u)=(1-t)^{-1}(1-u t)^{-1} P(t, u) \text { with } P \in \mathbb{Z}[t, u]
$$

\section{Furthermore:}

1) $\operatorname{deg}_{t} P=2 g$, $\operatorname{deg}_{u} P=g$.

2) In the expansion $P(t, u)=\sum_{i=0}^{2 g} P_{i}(u) t^{i}$ one has $P_{0}(u)=1$, $\operatorname{deg}_{u} P_{i}(u) \leq i / 2+1$ and $P_{2 g-i}(u)=u^{g-i} P_{i}(u)$ for $0 \leq i \leq 2 g$. 3) $P(1, u)=h$.

Here $\operatorname{deg}_{u}$ and $\operatorname{deg}_{t}$ denote the degree of a polynomial in the indicated variable. The above results are all taken from $[\mathbb{P}]$, Prop. 3.5 and we copied only those needed later on. Note that the statement $\operatorname{deg}_{u} P_{i}(u) \leq i / 2$ in [loc. cit.] is a misprint. Indeed, we will see below that one always has $\operatorname{deg}_{u} P_{1}(u)=1$ (unless $g=0$ ). As expected we have $P(t, u)=1$ in case $g=0$.

In [GS] G. van der Geer and R. Schoof used analogies from Arakelov-theory to define a two variable zeta-function for number fields along the above lines. As the number field case will serve only as a motivation in this note we refer to the original sources GS and [LR for definitions and to [D] for a 
comparison between them. Suffice it to say that in [LR], section 8 we find an entire function $\xi_{\mathbb{Q}}(w, s)$ of two complex variables which for $w=1$ equals Riemann's $\xi$-function. In particular, the zeroes of $\xi_{\mathbb{Q}}(1, s)$ are precisely the non-trivial zeros of the Riemann zeta-function. One is thus led to study the zero-locus of $\xi_{\mathbb{Q}}(w, s)$. J. Lagarias and E. Rains ask whether it might be the closure of a single irreducible complex-analytic variety of multiplicity one. The corresponding question in the geometric case seems to be whether the zero-locus of $P(t, u)$ is irreducible.

This is indeed the case:

Theorem 2 In the above situation, $P(t, u)$ is irreducible in $\mathbb{C}(u)[t]$.

As an illustration we discuss the cases $g=1$ and $g=2$ :

For $g=1$ setting $N:=\left|X\left(\mathbb{F}_{q}\right)\right|$ we have

$$
P(t, u)=1+(N-1-u) t+u t^{2}
$$

c.f. $[\mathrm{P}]$, example 3.4. This polynomial is reducible in $\mathbb{C}(u)[t]$ if and only if $N=0$ in which case we have $P(t, u)=(1-t)(1-u t)$. But it is well-known that a curve of genus one over a finite field always has a rational point, i.e. $N \neq 0$.

In case $g=2$ let the usual zeta-function of $X$ be

$$
Z(t)=(1-t)^{-1}(1-q t)^{-1} L(t)
$$

with

$$
L(t)=1+a t+b t^{2}+q a t^{3}+q^{2} t^{4}
$$

for certain $a, b \in \mathbb{Z}$. As $X$ is hyperelliptic, Prop. 4.3. of $\mathbb{P} \|$ can be used to compute

$P(t, u)=1+((a+q)-u) t+((q(q-1)+a q+b)-(a+q-1) u) t^{2}+((a+q)-u) u t^{3}+u^{2} t^{4}$.

This will not be used in the sequel and we omit the proof.

In order not to lead intuition astray we point out that in general $Z(t, u)$ is not determined by $Z(t)$, see $[\mathbb{P}]$, example 4.4 .

After a lengthy computation with discriminants one sees that a neccessary condition for this $P(t, u)$ to be reducible is

$$
b+a(q+1)+\left(q^{2}+1\right)=0 .
$$


But this expression equals $L(1)=h \neq 0$ !

The fact that $h \neq 0$ enters in the general proof of theorem 2 precisely through the condition $\beta \neq 0$ of lemma $\beta$ below. Note, however, that condition 2) of this lemma cannot be dropped. So one needs one more result on $P(t, u)$, contained in Prop. 4, which follows from Clifford's theorem.

I would like to thank C. Deninger for posing the above problem and for useful discussions on the topic.

\section{Proof}

We will use the following criterion for irreducibility:

Lemma 3 Let $k$ be a field, $F \in k[u, t]$ and assume:

1) $F$ is monic in $t$.

2) the leading coefficient of $F$ as a polynomial in $u$ is irreducible in $k[t]$.

3) there are $\alpha, \beta \in k, \beta \neq 0$ with $F(u, \alpha)=\beta$.

Then $F$ is irreducible in $k(u)[t]$.

This lemma will be applied to

$$
F=\tilde{P}(t, u):=t^{2 g} P\left(t^{-1}, u\right) \in \mathbb{C}[u, t] .
$$

Note that the irreducibility of $\tilde{P}$ in $\mathbb{C}(u)[t]$ will imply the irreducibility of $P$ because $P(0, u)=P_{0}(u)=1 \neq 0$ by theorem 11. The advantage of $\tilde{P}$ is that it is monic in $t$ and so satisfies condition 1) of lemma 3. Also 3) is satisfied (with $\alpha=1, \beta=h$ ) according to theorem 1, 3).

Proof (of lemma 3) Assume to the contrary that $F=f g$ in $k(u)[t]$ with $f$ and $g$ of positive degree and monic. One knows, c.f. for example $\mathbb{E}]$, Prop 4.11, that the coefficient of $f$ and $g$ are integral over $k[u]$ and as $k[u]$ is integrally closed we have $f, g \in k[u, t]$. So we can consider the decomposition $F=f g$ as polynomials in $u$ and infer from 2) that the leading coefficient of $f$ as a polynomial in $u$ lies in $k[t]^{*}=k^{*}$ (upon exchanging $f$ and $g$ if neccessary). In particular $n:=\operatorname{deg}_{u} f(u, t)=\operatorname{deg}_{u} f(u, \alpha)$. Substituting 3) 
gives $\beta=f(u, \alpha) g(u, \alpha)$ in $k[u]$. As $\beta \neq 0$ we get $n=0$, i.e. $f$ is constant in $u$ hence $f \in k^{*}$, contradiction.

We are left with verifying condition 2 ) of lemma 3 for the given $\tilde{P}$, i.e. the leading coefficient of $\tilde{P}$ as a polynomial in $u$ is irreducible in $k[t]$. We will in fact determine this coefficient:

Proposition 4 For $g \geq 1: \tilde{P}(t, u)=(1-t) u^{g}+O\left(u^{g-1}\right)$.

Proof We already know $\operatorname{deg}_{u} \tilde{P}=g$. Also the assertion is clear for $g=1$ from the formula for $P(t, u)$ recalled in the introduction. We assume $g \geq 2$. Looking at

$$
\tilde{P}(t, u)=t^{2 g}+P_{1}(u) t^{2 g-1}+\cdots+P_{g}(u) t^{g}+u P_{g-1}(u) t^{g-1}+\cdots+u^{g} t^{0}
$$

and using the bound $\operatorname{deg}_{u} P_{i}(u) \leq i / 2+1$ we see that $u^{g}$ can only occur in the last three terms: $u^{g-2} P_{2}(u) t^{2}+u^{g-1} P_{1}(u) t+u^{g}$. So the proof is completed by the following result on $P_{1}$ and $P_{2}$.

Proposition 5 For $g \geq 1$ :

1) $\operatorname{deg}_{u} P_{1}(u)=1$ and the leading coefficient is -1 .

2) $\operatorname{deg}_{u} P_{2}(u) \leq 1$.

Proof This is again clear for $g=1$. We assume $g \geq 2$ and write $P_{i}(u)=$ $\sum_{k} \alpha_{i k} u^{k}, \alpha_{i k} \in \mathbb{Z}$. As we already know $\operatorname{deg}_{u} P_{1}(u) \leq 1$ and $\operatorname{deg}_{u} P_{2}(u) \leq 2$ we need to show $\alpha_{11}=-1$ and $\alpha_{22}=0$. Recalling the notation $b_{n k}$ from the introduction we have the following

Claim: $b_{12}=\alpha_{00}+\alpha_{11}$ and $b_{23}=\alpha_{00}+\alpha_{11}+\alpha_{22}$.

Granting this we observe that Clifford's thorem, c.f. [H], IV, thm 5.4, gives $b_{12}=b_{23}=0$. Recalling also $\alpha_{00}=1$, because $P_{0}(u)=1$, and substituting gives indeed $\alpha_{11}=-1$ and $\alpha_{22}=0$. 
To prove the above claim we write the rational expression for $Z(t, u)$ in theorem 1 in terms of coefficients:

$$
Z(t, u)=\sum_{n \geq 0} \sum_{k \geq 1} b_{n k} \frac{u^{k}-1}{u-1} t^{n}=\left(\sum_{i \geq 0} t^{i}\right)\left(\sum_{j \geq 0}(u t)^{j}\right)\left(\sum_{l \geq 0} \sum_{k \geq 0} \alpha_{l k} u^{k} t^{l}\right) .
$$

This gives

\section{Lemma 6}

1) for $\nu, \alpha \geq 0: \sum_{k \geq \alpha+1} b_{\nu k}=\sum_{\mu, i \geq 0, \mu+i \leq \nu} \alpha_{i, \alpha-\mu}$

2) for $\nu \geq 0, \mu \geq 1$ : $b_{\nu \mu}=\sum_{i=0}^{\nu}\left(\alpha_{i, \mu-\nu-1+i}-\alpha_{i \mu}\right)$

We omit the details of this straightforward computation except to say that for 1 ) one uses $\frac{u^{k}-1}{u-1}=1+\cdots+u^{k-1}$ and 2) follows from 1) by a telescopesummation. In the formulation of the lemma it is understood that $\alpha_{n k}=0$ whenever $k<0$. We get from 2):

$$
b_{23}=\alpha_{00}-\alpha_{03}+\alpha_{11}-\alpha_{13}+\alpha_{22}-\alpha_{23}
$$

and

$$
b_{12}=\alpha_{00}-\alpha_{02}+\alpha_{11}-\alpha_{12} .
$$

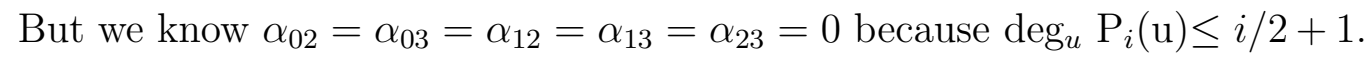
This concludes the proof of the claim, hence of theorem 2 .

\section{References}

[D] C. Deninger, A regularized product expansion for the two-variable zeta-function of van der Geer and Schoof, preprint, 2002.

[E] D. Eisenbud, Commutative Algebra with a View Towards Algebraic Geometry, GTM 150, Springer, 1995.

[GS] G. van der Geer, R. Schoof, Effectivity of Arakelov Divisors and the Theta Divisor of a Number Field, Selecta Math. (N.S.), 6, 2000, no. 4, pp 377-398. 
[H] R. Hartshorne, Algebraic Geometry, GTM 52, Springer, 1977.

[LR] J. Lagarias, E. Rains, On a Two-Variable Zeta Function for Number Fields, arXiv:math.NT/0104176 v5 7 Jul 2002.

[P] R. Pellikaan, On special divisors and the two variable zeta function of algebraic curves over finite fields, in: Arithmetic, Geometry and Coding Theory 4, Luminy, 1993, Walter de Gruyter, Berlin, 1996, pp 175-184.

Mathematisches Institut der WWU Münster

Einsteinstr. 62

48149 Münster

Germany

e-mail: naumannn@uni-muenster.de 